\title{
Perturbation of infinite networks of resistors
}

\author{
József Cserti ${ }^{1}$, Gyula Dávid ${ }^{2}$ and Attila Piróth ${ }^{3}$ \\ Eötvös University, \\ ${ }^{1}$ Department of Physics of Complex Systems, ${ }^{2}$ Department of Atomic Physics, \\ ${ }^{3}$ Department for Theoretical Physics, \\ H-1117 Budapest, Pázmány Péter sétány 1/A , Hungary
}

\begin{abstract}
The resistance between arbitrary nodes of infinite networks of resistors is studied when the network is perturbed by removing one bond from the perfect lattice. A connection is made between the resistance and the lattice Green's function of the perturbed network. Solving Dyson's equation the Green's function and the resistance of the perturbed lattice are expressed in terms of those of the perfect lattice. Numerical results are presented for a square lattice. Our method of the lattice Green's function in studying resistor networks can also be applied in the field of random walks as well as electrical and mechanical breakdown phenomena in insulators, thin films and modern ceramics.
\end{abstract}

\section{INTRODUCTION}

The falculation of the resistance between two arbitrary nodes of infinite networks of resistors is a well studied subject $t$. Recently, it has been demonstrated how the lattice Green's functions, as an alternative method to using the principle of the superposition of current distributions $\mathbf{3}$, can be applied to this problem $\mathbf{E}$. The interested readers will also find several useful references there.

The analytical behavior efthe lattice Green's function has been extensively studied in condensed matter physics over the past three decades 6 ; impurities are often modeled by a simple forms of perturbations (see Eq. (13) below). Thus, the resistor network problem can be successfully tackled by ifilizing the strong connection between the two fields, in particular through the use of the lattice Green's function $\mathbf{3}$. Our approach may also serve as a didactic introduction of the Green's function method used in solid state physics as well as field theory. The perturbation series that show up in these fields are, in general, infinite and need to undergo a tedious renormalization procedure. In contrast, the perturbative solutions of the Dyson equation can be summed up in closed form in our case, due to the special form (13) of the perturbation. Studying the analytical behavior of the closed-form Green's function has also proven pedagogically useful in introductory courses on solid state physics and field theory.

The conductivity of lattices with randomly distributed defects has also been thoroughly investigated in the past 25 years within the framework of effective medium theories 8 . method 10 . Below, we shall use the Green's function approach to study a special case, i.e. to determine the resistance for a so-called perturbed lattice that is obtained by removing one bond from the perfect lattice (corresponding to "simple perturbation" mentioned in the previous paragraph). Although this system is much less complex than those studied previously, it has the great advantage of being analytically treatable and leading to closed-form expressions for the resistances.

As an example (see Fig. 1), consider an infinite square lattice whose edges represent identical resistances $R$. Removing one edge (bond) from this perfect lattice results in a perturbed lattice.

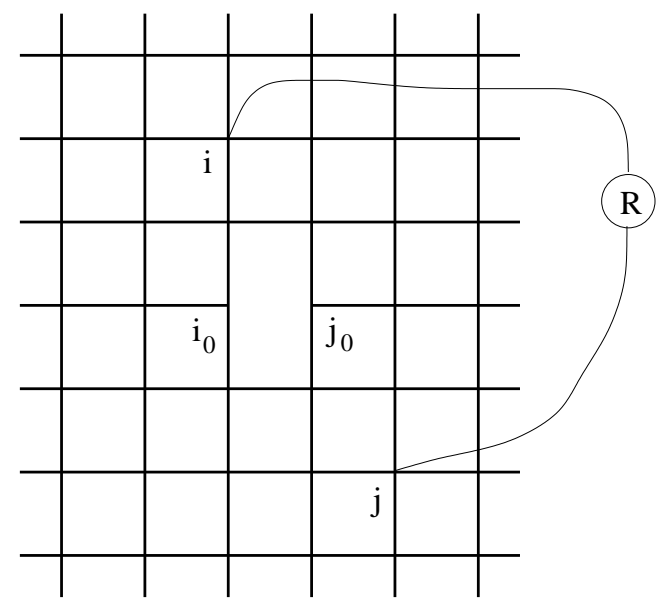


FIG. 1. Perturbation of an infinite square lattice by removing one edge between sites $\mathbf{r}_{i_{0}}$ and $\mathbf{r}_{j_{0}}$. Each edge represents a resistance $R$. The resistance $R(i, j)$ is measured between arbitrary lattice points $\mathbf{r}_{i}$ and $\mathbf{r}_{j}$.

Then, one can ask the resistance between two arbitrary nodes. It is simple to find the resistance between the two

ends of the missing bond. On one hand it is well known (see e.g. Aitchison's papen11) that for a perfect square lattice the resistance between adjacent nodes is $R / 2$. On the other hand this resistance equals the parallel resultant of $R$ and the resistance we wish to find. Thus, it is obvious that the resistance between the two ends of the missing bond in the perturbed lattice is $R$.

Finding the resistance between two arbitrary nodes in the perturbed lattice is much more difficult. In this paper we present a further application of the Green's function method 5 to answer this question. Our treatment of the problem is based on the idea used by Kirkpatrick目 for studying the transport in inhomogeneous conductors. The Dirac notation of the vectors is used throughout this paper. Note that the derivation and our formulas presented below can be straightforwardly expressed by the usual matrix-element formalism. However, the Dirac notation seems to provide the most powerful and elegant formulation of the problem. The readers who are unfamiliar with this formalism will find sufficient background material in several well-known textbooks, e.g. Schwabl12.

According to Ohm's and Kirchhoff's laws, for a given current distribution, the potentials on the lattice sites are given by a Poisson-like equation involving a so-called lattice Laplacian operator. This operator can be decomposed into two parts: one associated with the perfect lattice and the other corresponding to the perturbation. A relation between the resistance and the Green's function for the perturbed lattice is derived. The problem thus reduces to the calculation of the Green's function for the perturbed lattice which actually satisfies the Dyson equation 6 . As we shall see, the Dyson equation can be solved exactly in the present problem. Then, an explicit formula can be derived for the resistance in the perturbed lattice in terms of the resistances between different nodes in the perfect lattice. Our Green's function method proved to be a highfoffective technique and can successfully tackle the present problem even in cases when the superposition principle H $^{-}$faces hardly (or even in-)surmountable difficulties.

\section{GREEN'S FUNCTION AND RESISTANCE FOR PERFECT LATTICE}

To establish the method and the notations that we shall use throughout this paper, the case of a perfect lattice is reviewed first. Consider a perfect $d$-dimensional infinite lattice made up of identical resistances $R$. All lattice points are specified by position vectors $\mathbf{r}$ given in the form

$$
\mathbf{r}=l_{1} \mathbf{a}_{1}+l_{2} \mathbf{a}_{2}+\cdots+l_{d} \mathbf{a}_{d}
$$

where $\mathbf{a}_{1}, \mathbf{a}_{2}, \cdots, \mathbf{a}_{d}$ are independent primitive translation vectors, and $l_{1}, l_{2}, \cdots, l_{d}$ range through all integer values. We denote the potential at site $\mathbf{r}_{i}$ and the current entering at lattice point $\mathbf{r}_{i}$ by $V\left(\mathbf{r}_{i}\right)$ and $I\left(\mathbf{r}_{i}\right)$, respectively. According to Ohm's and Kirchhoff's laws, we may write

$$
\sum_{\mathbf{n}}\left[V\left(\mathbf{r}_{i}\right)-V\left(\mathbf{r}_{i}+\mathbf{n}\right)\right]=R I\left(\mathbf{r}_{i}\right),
$$

where the $\mathbf{n}$ are the vectors from site $\mathbf{r}_{i}$ to its nearest neighbors. One can form two vectors, $V$ and $I$ from the potentials and the currents at sites $\mathbf{r}_{i}$ :

$$
\begin{gathered}
V=\sum_{i}|i\rangle V_{i}, \\
I=\sum_{i}|i\rangle I_{i}
\end{gathered}
$$

where $V_{i}=V\left(\mathbf{r}_{i}\right)$ and $I_{i}=I\left(\mathbf{r}_{i}\right)$. Here it is assumed that $|i\rangle$, associated with the site $\mathbf{r}_{i}$, forms a complete orthonormal set, i.e. $\langle i \mid k\rangle=\delta_{i k}$ and $\sum_{i}|i\rangle\langle i|=1$. Using the vectors $V$ and $I$, Eq. (2) can be rewritten as

$$
\sum_{j}\left(z \delta_{i j}-\Delta_{i j}\right)\langle j| V=R\langle i| I,
$$

where $z$ is the number of neighbors (coordination number) of each lattice site (e.g. $z=2 d$ for a $d$-dimensional hypercubic lattice), and $\Delta_{k l}$ is unity if the sites $\mathbf{r}_{k}$ and $\mathbf{r}_{l}$ are nearest neighbors and zero otherwise. The summation is taken over all lattice sites. Premultiplying both sides of the above equation by $|i\rangle$ and summing over $i$, we have 


$$
L_{0} V=-R I,
$$

where $L_{0}$ is the so-called lattice Laplaciant:

$$
L_{0}=\sum_{i, j}|i\rangle\left(\Delta_{i j}-z \delta_{i j}\right)\langle j| .
$$

For further progress it is useful to define the lattice Green's function of $L_{0}$. Similarly to the common definition used in the literature 6 , the lattice Green's function is defined by

$$
L_{0} G_{0}=-1 .
$$

The properties of this lattice Green's function and its explicit form in coordinate representation are given in Appendix A. The solution of the Poisson-like Eq. (6) can then be given in a simple form:

$$
V=-R L_{0}^{-1} I=R G_{0} I .
$$

To measure the resistance between two arbitrary sites we assume that a current $I_{0}$ enters at site $\mathbf{r}_{i}$ and a current $-I_{0}$ exits at site $\mathbf{r}_{j}$, while the currents are zero at all other lattice points. The current distribution may thus be written as

$$
I_{m}=I_{0}\left(\delta_{m i}-\delta_{m j}\right) \text { for all } \mathrm{m} .
$$

The potentials at different sites can be determined by inserting the above-given current distribution into Eq. (9), and one finds

$$
V_{k}=\langle k| V=R\langle k| G_{0} I=R \sum_{m}\left\langle k\left|G_{0}\right| m\right\rangle I_{m}=R I_{0}\left[G_{0}(k, i)-G_{0}(k, j)\right]
$$

where $G_{0}(n, m)=\left\langle n\left|G_{0}\right| m\right\rangle$ is the matrix element of the operator $G$ in the basis $|n\rangle$. The resistance between sites $\mathbf{r}_{i}$ and $\mathbf{r}_{j}$ is then

$$
R_{0}(i, j)=\frac{V_{i}-V_{j}}{I_{0}}=2 R\left[G_{0}(i, i)-G_{0}(i, j)\right]
$$

where we have made use of the symmetry properties of the Green's function $G_{0}(i, j)$ given in Appendix A. The same expression for the resistance in terms of the lattice Green's function was derived in Ref. $D$.

\section{GREEN'S FUNCTION AND RESISTANCE FOR PERTURBED LATTICE. DYSON'S EQUATION}

The current contribution $\delta I_{i}$ at site $\mathbf{r}_{i}$ due to the bond $\left(i_{0} j_{0}\right)$ is given by

$$
\begin{aligned}
\delta I_{i} R & =\delta_{i i_{0}}\left(V_{i_{0}}-V_{j_{0}}\right)+\delta_{i j_{0}}\left(V_{j_{0}}-V_{i_{0}}\right) \\
& =\left\langle i \mid i_{0}\right\rangle\left(\left\langle i_{0}\right|-\left\langle j_{0}\right|\right) V+\left\langle i \mid j_{0}\right\rangle\left(\left\langle j_{0}\right|-\left\langle i_{0}\right|\right) V \\
& =\left\langlei \left|\left(\left|i_{0}\right\rangle-\left|j_{0}\right\rangle\right)\left(\left\langle i_{0}\right|-\left\langle j_{0}\right|\right) V=\langle i| L_{1} V,\right.\right.
\end{aligned}
$$

where the operator $L_{1}$ is of a so-called 'dyadic' form:

$$
L_{1}=\left(\left|i_{0}\right\rangle-\left|j_{0}\right\rangle\right)\left(\left\langle i_{0}\right|-\left\langle j_{0}\right|\right) .
$$

Here we used the fact that $\delta_{n m}=\langle n \mid m\rangle$. Removing this bond from the perfect lattice the current $I_{i}$ at site $\mathbf{r}_{i}$ is given by

$$
\left(-L_{0} V\right)_{i}-R \delta I_{i}=R I_{i} .
$$

Thus, Ohm's and Kirchhoff's laws for the perturbed lattice can be written as

$$
\begin{aligned}
L V & =-R I, \quad \text { where } \\
L & =L_{0}+L_{1} .
\end{aligned}
$$


Equation (15) is similar to that found in the case of a perfect lattice. However, the operator $L$ is now a sum of $L_{0}$ associated with the perfect lattice and a 'perturbation' given by $L_{1}$. Note that the same decomposition of the Laplacian operator with the perturbation $L_{1}$ given by (13) was used by Kirkpatrick.

Similarly to the perfect lattice, the Green's function $G$ for the perturbed lattice is defined by

$$
L G=-1
$$

The resistance for the perturbed lattice can be obtained in terms of the Green's function much in the same way as for a perfect lattice. Measuring the resistance between sites $\mathbf{r}_{i}$ and $\mathbf{r}_{j}$, we assume that a current $I_{0}$ enters at site $\mathbf{r}_{i}$ and a current $-I_{0}$ exits at $\mathbf{r}_{j}$. The currents are zero at all other lattice points. Thus, the current distribution is $I_{m}=I_{0}\left(\delta_{m i}-\delta_{m j}\right)$ for all $m$. From Eqs. (15) and (17) we have $V_{k}=\langle k| V=R\langle k| G I=R\left\langle k\left|G \sum_{m}\right| m\right\rangle I_{m}=$ $R \sum_{m}\langle k|G| m\rangle I_{m}=R \sum_{m} G(k, m) I_{m}$. Inserting the current distribution $I_{m}$ one finds

$$
V_{k}=R I_{0}[G(k, i)-G(k, j)] .
$$

Therefore, the resistance between sites $\mathbf{r}_{i}$ and $\mathbf{r}_{j}$ reads

$$
R(i, j)=\frac{V_{i}-V_{j}}{I_{0}}=R[G(i, i)-G(i, j)+G(j, j)-G(j, i)] .
$$

Note that unlike for a perfect lattice, $G(i, i) \neq G(j, j)$ since translational symmetry is broken in the perturbed lattice. However, as we shall see, $G(i, j)=G(j, i)$. The problem of finding the resistance reduces to the calculation of the Green's function for the perturbed lattice.

Perturbation theory for the Green's function is worked out in the literature (see e.g. Economou's book 6 ). Using Eqs. (8), (16) and (17) yields $\left(-G_{0}^{-1}+L_{1}\right) G=-1$. Premultiplying both sides of this equation by $G_{0}$ one obtains the so-called Dyson's equation:

$$
G=G_{0}+G_{0} L_{1} G
$$

This is an equation for $G$ in terms of $G_{0}$ (which is assumed to be known), and the perturbation $L_{1}$. In general, the solution of the Dyson equation can be found by iteration resulting in an infinite series:

$$
G=G_{0}+G_{0} L_{1} G_{0}+G_{0} L_{1} G_{0} L_{1} G_{0}+\cdots .
$$

However, if $L_{1}$ has a special form - such as that given in Eq. (13) - the Dyson equation can be solved exactly. Now the perturbation $L_{1}$ is equal to the dyadic product of the vector $\left|i_{0}\right\rangle-\left|j_{0}\right\rangle$ with itself. In this case one may apply the identity

$$
(A+|x\rangle\langle y|)^{-1}=A^{-1}-\frac{A^{-1}|x\rangle\langle y| A^{-1}}{1+\left\langle y\left|A^{-1}\right| x\right\rangle}
$$

valid for arbitrary vectors $|x\rangle$ and $|y\rangle$ whose dimensions are the same as that of operator $A$, assuming that the inverse of operator $A$ exists and the denominator $1+\left\langle y\left|A^{-1}\right| x\right\rangle \neq 0$. This identity is readily proved by postmultiplying Eq. (22) with $A+|x\rangle\langle y|$. Using the above identity with $A=L_{0},|x\rangle=\left|i_{0}\right\rangle-\left|j_{0}\right\rangle$ and $\langle y|=\left\langle i_{0}\right|-\left\langle j_{0}\right|$ one obtains for the Green's function:

$$
G=-\left(L_{0}+L_{1}\right)^{-1}=G_{0}+\frac{G_{0}\left(\left|i_{0}\right\rangle-\left|j_{0}\right\rangle\right)\left(\left\langle i_{0}\right|-\left\langle j_{0}\right|\right) G_{0}}{1-2\left[G_{0}\left(i_{0}, i_{0}\right)-G_{0}\left(i_{0}, j_{0}\right)\right]} .
$$

Below we shall see that the denominator in Eq. (23) is never equal to zero when $d>1$. The matrix elements of the operator $G$ can then be expressed with the matrix elements of $G_{0}$ as

$$
G(i, j)=\langle i|G| j\rangle=G_{0}(i, j)+\frac{\left[G_{0}\left(i, i_{0}\right)-G_{0}\left(i, j_{0}\right)\right]\left[G_{0}\left(i_{0}, j\right)-G_{0}\left(j_{0}, j\right)\right]}{1-2\left[G_{0}\left(i_{0}, i_{0}\right)-G_{0}\left(i_{0}, j_{0}\right)\right]} .
$$

There is an alternative way to calculate $G$. This method is analogous to that presented in Chapter 6 . of Economou's book 9 for the calculation of the Green's function. There the system was condensed matter modeled by a periodic tight-binding Hamiltonian with one impurity given by Dirac delta potential.

One can insert $L_{1}$ given by (13) into the series in (21). Then the matrix elements of $G$ result in infinite geometric series, which can be summed up exactly leading to the same results as above. 
Note that this geometric series as well as the perturbative solution of the Dyson equation given by Eq. (21) is not necessarily convergent, especially in the case of dyadic perturbations with $\left|\left\langle y\left|A^{-1}\right| x\right\rangle\right| \geq 1$. However, Eq. (22) is still valid even for $\left|\left\langle y\left|A^{-1}\right| x\right\rangle\right|>1$.

It is clear from $G_{0}(i, j)=G_{0}(j, i)$ that $G(i, j)$ is also a symmetric matrix, i.e. $G(i, j)=G(j, i)$. ¿From Eqs. (19) and (24) the resistance between $\mathbf{r}_{i}$ and $\mathbf{r}_{j}$ can be obtained in terms of the matrix elements of $G_{0}$ :

$$
\begin{aligned}
\frac{R(i, j)}{R} & =G(i, i)+G(j, j)-2 G(i, j) \\
& =2\left[G_{0}(i, i)-G_{0}(i, j)\right]+\frac{\left[G_{0}\left(i, i_{0}\right)-G_{0}\left(i, j_{0}\right)-G_{0}\left(j, i_{0}\right)+G_{0}\left(j, j_{0}\right)\right]^{2}}{1-2\left[G_{0}\left(i_{0}, i_{0}\right)-G_{0}\left(i_{0}, j_{0}\right)\right]} .
\end{aligned}
$$

Using Eq. (12) this formula can be rewritten in terms of the resistances in a perfect lattice:

$$
R(i, j)=R_{0}(i, j)+\frac{\left[R_{0}\left(i, j_{0}\right)+R_{0}\left(j, i_{0}\right)-R_{0}\left(i, i_{0}\right)-R_{0}\left(j, j_{0}\right)\right]^{2}}{4\left[R-R_{0}\left(i_{0}, j_{0}\right)\right]} .
$$

This is our final result for the resistance between two arbitrary nodes $\mathbf{r}_{i}$ and $\mathbf{r}_{j}$ of the perturbed lattice in which the bond $\left(i_{0} j_{0}\right)$ is removed.

It is easy to calculate the resistance between sites $\mathbf{r}_{i_{0}}$ and $\mathbf{r}_{j_{0}}$ for a $d$-dimensional hypercubic lattice. For symmetry reasons 5 the resistance between $\mathbf{r}_{i_{0}}$ and $\mathbf{r}_{j_{0}}$ in a perfect lattice is $R_{0}\left(i_{0}, j_{0}\right)=R / d$ if $d>1$. Then, using Eq. (26), the resistance between the two ends of the removed bond is $R\left(i_{0}, j_{0}\right)=R /(d-1)$. For a square lattice $(d=2)$ the resistance is $R$ as mentioned in the introduction. It also follows that the denominator in Eq. (26) is never equal to zero, and this is necessarily true for each previous equation that contains this term.

Note that the explicit form of the lattice Laplacian $L_{0}$ defined in Eq. (7) was not used in the derivation of Eq. (26). Thus, our final result for the resistance in the perturbed lattice is valid for any lattice structure in which each unit cell has only one lattice site. This is the case, for example, for triangular lattices.

Finally, it is worth mentioning some possible applications of our method and results. In Doyle and Snell's book the connection between random walks and electric networks is presented. Thus, our result can also be extended to the random walk problem in a perturbed lattice. Furthermore, the highly effective method of the lattice Green's function in studying resistor networks also provides a pedagogically useful way to familiarize students with the notions of the Dyson equation and the Green's function.

When more than one bond is missing from the perfect lattice, our method (outlined above) can be iterated. It can be shown that our final result (26) is still valid provided the values of $R_{0}$ of the perfect lattice are replaced by the values of the resistances obtained from the previous step of the iteration. Thus, lattices with more complex defects can be studied analytically with our method. An example is the so-called crack-type defects arising in-several fields such as electrical and mechanical breakdown phenomena in insulators, thin films and modern ceramics.13. The current distribution around cracks can be easily calculated from the Green's function. This problem was studied numerically as well as analytically in the continuum limit (for a recent paper see e.g. Boksiner and Leath ${ }^{14}$ and references therein). Application of our method to investigate this problem is in progress.

\section{NUMERICAL RESULTS}

Below we shall present some numerical results for square lattices. Recurrence relations for the resistances between arbitrary nodes were derived for perfect square lattices in Ref.E. These recurrence formulas provide a very simple and effective tool for the calculation of the resistance. For convenience, we invoke them:

$$
\begin{aligned}
R_{0}(m+1, m+1) & =\frac{4 m}{2 m+1} R_{0}(m, m)-\frac{2 m-1}{2 m+1} R_{0}(m-1, m-1), \\
R_{0}(m+1, m) & =2 R_{0}(m, m)-R_{0}(m, m-1), \\
R_{0}(m+1,0) & =4 R_{0}(m, 0)-R_{0}(m-1,0)-2 R_{0}(m, 1), \\
R_{0}(m+1, p) & =4 R_{0}(m, p)-R_{0}(m-1, p)-R_{0}(m, p+1)-R_{0}(m, p-1) \text { if } 0<p<m,
\end{aligned}
$$

where the indices $m$ and $n$ of $R_{0}(m, n)$ (not to be confused with $i$ and $j$ above) stand for the relative coordinates of the two sites on the square lattice. Since the exact values of $R_{0}(1,0)=R / 2$ and $R_{0}(1,1)=2 R / \pi$ are known (obviously 
$\left.R_{0}(0,0)=0\right)$, one can calculate the resistance exactly for arbitrary nodes by using the above recurrence formulas. It is interesting to note that using these formulas one has, in units of $R$

$$
R_{0}(50,50)=\frac{6400711399252571342562758751832284129928}{1089380862964257455695840764614254743075} \frac{1}{\pi} \approx 1.87025,
$$

which can be found quickly (within a few second) using the program MAPLE. In Fig. 2 the resistance $R_{0}(i, j)$ between the node $i=(0,0)$ and $j=\left(j_{x}, j_{y}\right)$ is plotted as a function of $j_{x}$ and $j_{y}$ for a perfect square lattice.

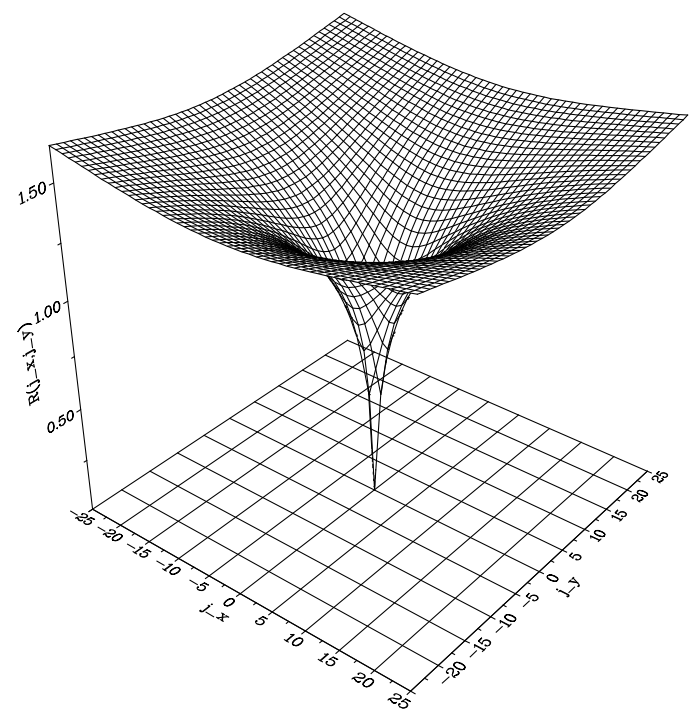

FIG. 2. The resistance $R_{0}(i, j)$ between nodes $i=(0,0)$ and $j=\left(j_{x}, j_{y}\right)$ as a function of $j_{x}$ and $j_{y}$ for a perfect square lattice.

One can see from the figure that by increasing the distance between the nodes $i$ and $j$ the rotational symmetry of the resistance is more and more recovered. Indeed, in Ref. It $_{\text {it }}$ was shown that for large values of $j_{x}$ or/and $j_{y}$, the asymptotic form of the resistance is

$$
R\left(j_{x}, j_{y}\right)=\frac{R}{\pi}\left(\ln \sqrt{j_{x}^{2}+j_{y}^{2}}+\gamma+\frac{\ln 8}{2}\right),
$$

where $\gamma=0.5772 \ldots$ is the Euler-Mascheroni constant.

On the perturbed square lattice the resistance can be calculated from Eq. (26). As an example we show results when site $\mathbf{r}_{i}$ is fixed and $\mathbf{r}_{j}$ is moved along the line of the removed bond. The resistance is measured between nodes $\mathbf{r}_{i}$ and $\mathbf{r}_{j}$, where $\mathbf{r}_{i}=(0,0)$ and $\mathbf{r}_{j}=\left(j_{x}, 0\right)$. The two ends of the removed bond are $\mathbf{r}_{i_{0}}=(0,0)$ and $\mathbf{r}_{j_{0}}=(1,0)$. In Fig. 3 the resistances for the perfect and the perturbed lattices are plotted as functions of $j_{x}$. 


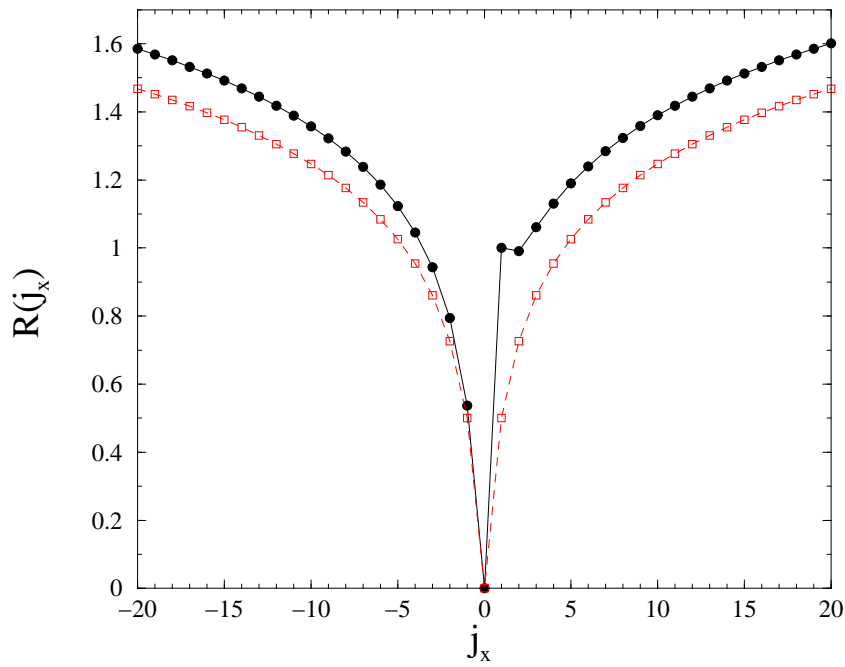

FIG. 3. In an infinite square lattice the origin of the coordinate system is at site $\mathbf{r}_{i}$, i.e. $\mathbf{r}_{i}=(0,0)$. The ends of the removed bond are at $\mathbf{r}_{i_{0}}=(0,0)$ and $\mathbf{r}_{j_{0}}=(1,0)$. The resistances on the perturbed and the perfect square lattices are measured between sites $\mathbf{r}_{i}$ and $\mathbf{r}_{j}$, where $\mathbf{r}_{j}=\left(j_{x}, 0\right)$. They are plotted as functions of $j_{x}$ (filled circles and empty squares, respectively). The solid and dashed lines are just for guiding the eyes.

One can see that the resistance is always larger in the perturbed lattice than in the perfect lattice. This intuitively obvious fact follows from the positivity of the second term in Eq. (26). It can also be seen from the figure that increasing the distance between nodes $\mathbf{r}_{i}$ and $\mathbf{r}_{j}$ the resistance tends to that of the perfect lattice. The resistance is not symmetric under the transformation $j_{x} \rightarrow-j_{x}$ because translational symmetry is broken in the perturbed lattice.

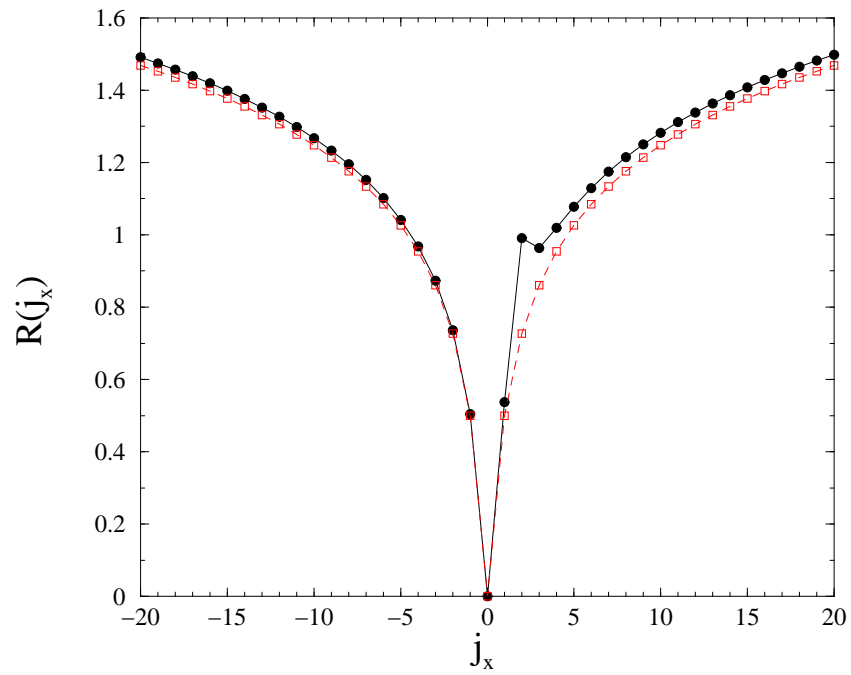

FIG. 4. The same as Fig. 3 except that the ends of the removed bond are at $\mathbf{r}_{i_{0}}=(1,0)$ and $\mathbf{r}_{j_{0}}=(2,0)$.

The configuration is the same in Fig. 1 as in Fig. 3 except that $\mathbf{r}_{i_{0}}=\mathbf{r}_{i}+(1,0)$. Similar features are observed in the two cases. However, the resistance on the perturbed lattice tends to that of the perfect lattice more rapidly in Fig. 4 than in Fig. 3 as $j_{x}$ increases.

Finally, it worth mentioning that a similar analysis of the resistance can be carried out for simple cubic lattices. Recently, Glasser et al. 15 have expressed the exact values for the cubic lattice Green's functions in terms of gamma functions. Using their results one can calculate the resistance for perfect simple cubic lattices and for perturbed lattices. 


\section{ACKNOWLEDGMENTS}

The authors wish to thank S. Redner, L. Glasser and P. Gnädig for helpful discussions. This work was supported by the EU. RTN within the programme "Nanoscale Dynamics, Coherence and Computation", and the Hungarian Science Foundation OTKA TO25866 and TO34832.

\section{APPENDIX A: EXPLICIT FORM OF THE LATTICE GREEN'S FUNCTIONS FOR PERFECT LATTICE STRUCTURES}

In this section we shall present the symmetry properties of the lattice Green's function $G_{0}$, and derive its explicit form in coordinate representation for perfect lattice structures.

From Eq. (7) we have $L_{0}(i, j)=\left\langle i\left|L_{0}\right| j\right\rangle=L_{0}(j, i)$. Therefore,

$$
G_{0}(i, j)=\left\langle i\left|G_{0}\right| j\right\rangle=G_{0}(j, i) .
$$

Thus, $G_{0}(i, j)$ is a symmetric matrix. The perfect lattice is translationally invariant, so $G_{0}(i, j)$ depends only on the relative coordinates of sites $\mathbf{r}_{i}$ and $\mathbf{r}_{j}$. Thus we have

$$
G_{0}(i, i)=G_{0}(j, j) .
$$

Let us turn to the derivation of the coordinate representation of $G_{0}$. Consider a finite part of the lattice constructed by repeating the unit cell (say $N_{i}$ times) along the unit vectors $\mathbf{a}_{i}$, where $i=1,2, \cdots, d$. Define the Fourier transform of the basis $|i\rangle$ by

$$
\mid \mathbf{k})=\frac{1}{\sqrt{N}} \sum_{i} e^{i \mathbf{k r}_{i}}|i\rangle
$$

The lattice sites $\mathbf{r}_{i}$ are specified by Eq. (11), and the vectors $\mathbf{k}$ belonging to the first Brillouin zone (BZ) are given by

$$
\mathbf{k}=\frac{m_{1}}{N_{1}} \mathbf{b}_{1}+\frac{m_{2}}{N_{2}} \mathbf{b}_{2}+\cdots+\frac{m_{d}}{N_{d}} \mathbf{b}_{d}
$$

where $m_{1}, m_{2}, \cdots m_{d}$ are integers such that $-N_{i} / 2 \leq m_{i} \leq N_{i} / 2$ for $i=1,2, \cdots, d$, and the $\mathbf{b}_{j}$ are the reciprocal lattice vectors defined by $\mathbf{a}_{i} \mathbf{b}_{j}=2 \pi \delta_{i j}, i, j=1,2, \cdots, d$. The total number of lattice sites is $N=N_{1} N_{2} \cdots N_{d}$. Periodic boundary conditions are assumed at the boundary of the finite lattice, and in the final results the limit $N \rightarrow \infty$ is taken. It is also assumed that $N_{i}$ is an even integer, which will be irrelevant in the limit $N \rightarrow \infty$. The mathematical description of crystal lattices and the concept of the Brillouin zone can be found in many books on solid state physic $\mathbf{1 6}$. To distinguish the basis vectors $|i\rangle$ from their Fourier transforms $\mid \mathbf{k}$ ), parentheses will be used instead of angled brackets. Using the so-called lattice summation rules $1 \mathrm{E}$

$$
\begin{aligned}
& \sum_{i} e^{i\left(\mathbf{k}-\mathbf{k}^{\prime}\right) \mathbf{r}_{i}}=N \delta_{\mathbf{k} \mathbf{k}^{\prime}} \\
& \sum_{\mathbf{k}} e^{i \mathbf{k}\left(\mathbf{r}_{i}-\mathbf{r}_{j}\right)}=N \delta_{i j},
\end{aligned}
$$

it can be shown that the basis $\mid \mathbf{k})$ is a complete orthonormal set, i.e. $\left(\mathbf{k} \mid \mathbf{k}^{\prime}\right)=\delta_{\mathbf{k k}^{\prime}}$ and $\left.\sum_{\mathbf{k}} \mid \mathbf{k}\right)(\mathbf{k} \mid=1$ provided the basis $|i\rangle$ is complete and orthonormal. It is also clear that

$$
\langle i| \mathbf{k})=\frac{1}{\sqrt{N}} e^{i \mathbf{k r}_{i}}
$$

The Laplacian operator $L_{0}$ for a perfect lattice is a diagonal matrix in the basis $\left.\mid \mathbf{k}\right)$ since

$$
\left(\mathbf{k}\left|L_{0}\right| \mathbf{k}^{\prime}\right)=\sum_{i j}\left(\mathbf{k}|i\rangle\left(\Delta_{i j}-z \delta_{i j}\right)\langle j| \mathbf{k}^{\prime}\right)=\frac{1}{N} \sum_{i j} e^{-i \mathbf{k} \mathbf{r}_{i}}\left(\Delta_{i j}-z \delta_{i j}\right) e^{-i \mathbf{k}^{\prime} \mathbf{r}_{j}}=-\varepsilon(\mathbf{k}) \delta_{\mathbf{k k}^{\prime}},
$$

where 


$$
\varepsilon(\mathbf{k})=z-\sum_{\mathbf{n}} e^{i \mathbf{k n}}
$$

and vectors $\mathbf{n}$ point from some lattice point into its the nearest neighbors.

In coordinate representation the matrix element of the Green's function $G_{0}$ can be written as

$$
\left.G_{0}(i, j)=\left\langle i\left|G_{0}\right| j\right\rangle=\sum_{\mathbf{k} \mathbf{k}^{\prime}}\langle i| \mathbf{k}\right)\left(\mathbf{k}\left|G_{0}\right| \mathbf{k}^{\prime}\right)\left(\mathbf{k}^{\prime}|i\rangle=\frac{1}{N} \sum_{\mathbf{k} \mathbf{k}^{\prime}}\left(\mathbf{k}\left|G_{0}\right| \mathbf{k}^{\prime}\right) e^{i\left(\mathbf{k r}_{i}-\mathbf{k}^{\prime} \mathbf{r}_{j}\right)}\right.
$$

In the third step the completeness of the basis $\mid \mathbf{k})$ has been used. Since $G_{0}=-L_{0}$, one finds

$$
G_{0}(i, j)=\frac{1}{N} \sum_{\mathbf{k} \in \mathrm{BZ}} \frac{e^{i \mathbf{k}\left(\mathbf{r}_{i}-\mathbf{r}_{j}\right)}}{\varepsilon(\mathbf{k})}
$$

Note that the toroidal and cylindrical geometry cases discussed by Jeng 17 can be straightforwardly treated by direct summation over $\mathbf{k}$. For infinite lattices (in the limit $N \rightarrow \infty$ ) the discrete $\mathbf{k}$ summation can be substituted by an integral16:

$$
\sum_{\mathbf{k} \in \mathrm{BZ}} \rightarrow \frac{N v_{0}}{(2 \pi)^{d}} \int_{\mathbf{k} \in \mathrm{BZ}} d^{d} \mathbf{k}
$$

where $v_{0}$ is the volume of the unit cell of the lattice. Thus, the lattice Green's function in coordinate representation becomes

$$
G_{0}(i, j)=v_{0} \int_{\mathbf{k} \in \mathrm{BZ}} \frac{d^{d} \mathbf{k}}{(2 \pi)^{d}} \frac{e^{i \mathbf{k}\left(\mathbf{r}_{i}-\mathbf{r}_{j}\right)}}{\varepsilon(\mathbf{k})} .
$$

One can see that $G_{0}(i, j)$ depends only on the relative coordinates of sites $\mathbf{r}_{i}$ and $\mathbf{r}_{j}$, as stated in Eq. (A2). For example, $z=2 d$ for the $d$-dimensional hypercubic lattice, and one finds $\varepsilon(\mathbf{k})=2\left(d-\sum_{i=1}^{d} \cos \mathbf{k a}_{i}\right)$. For body centered cubic and face centered cubic lattice structures see eg. the introduction of the lattice Green's function by Katsura et ald, and for triangular and hexagonal lattices Ref.5.

${ }^{1}$ B. van der Pol and H. Bremmer, Operational Calculus Based on the Two-Sided Laplace Integral (Cambridge University Press, England, 1955) 2nd ed., p. 372.

${ }^{2}$ P. G. Doyle and J. L. Snell, Random Walks and Electric Networks, (The Carus Mathematical Monograph, series 22, The Mathematical Association of America, USA, 1984) pp. 83-149.

${ }^{3}$ G. Venezian, "On the resistance between two points on a grid," Am. J. Phys. 62, 1000-1004 (1994).

${ }^{4}$ D. Atkinson and F. J. van Steenwijk, "Infinite resistive lattices," Am. J. Phys. 67, 486-492 (1999).

${ }^{5}$ J. Cserti, "Application of the lattice Green's function for calculating the resistance of an infinite network of resistors," Am. J. Phys. 68, 896-906 (2000).

${ }^{6}$ E. N. Economou, Green's functions in Quantum Physics (Springer-Verlag, Berlin, Germany, 1983), 2nd ed.

${ }^{7}$ S. Katsura, T. Morita, S. Inawashiro, T. Horiguchi, and Y. Abe, "Lattice Green's Function. Introduction," J. Math. Phys. 12, 892-895 (1971).

${ }^{8}$ S. Kirkpatrick, "Percolation and Conduction," Rev. Mod. Phys. 45, 574-588 (1973).

9 J. Koplik, "On the effective medium theory of random linear networks," Journal of Physics C: Solid State Physics 14, 4821-4837 (1981).

${ }^{10}$ S. Redner, "Conductivity of random resistor-diode networks," Phys. Rev. B 25, 5646-5655 (1982).

${ }^{11}$ R. E. Aitchison, "Resistance between adjacent points of Liebman mesh," Am. J. Phys. 32, 566 (1964).

${ }^{12}$ F. Schwabl, Quantum Mechanics (Springer-Verlag, Berlin, Germany, 1988).

${ }^{13}$ P. M. Duxbury, P. L. Leath and P. D. Beale, "Breakdown properties of quenched random systems: The random-fuse network," Phys. Rev. B 36, 367-380 (1987).

14 J. Boksiner and P. L. Leath, "Dielectric breakdown in media with defects," Phys. Rev. E57, 3531-3541 (1998).

${ }^{15}$ M. L. Glasser and J. Boersma, "Exact values for the cubic lattice Green functions," J. Phys. A: Math. Gen. 33, 5017-5023 (2000). 
${ }^{16}$ P. M. Chaikin and T. C. Lubensky, Principles of Condensed Matter Physics (Cambridge University Press, Cambridge, England, 1995), chapter 2.; J. M. Ziman, Principles of The Theory of Solids (Cambridge University Press, Cambridge, England, 1972), chapter 1; N. W. Ashcroft and N. D. Mermin, Solid State Physics (Saunders College, Philadelphia, PA, 1976), chapter 4.; C. Kittel, Introduction to Solid State Physics (John Wiley and Sons, New York, 1986), 6th ed., pp. 37-42.

${ }^{17}$ M. Jeng, "Random walks and effective resistances on toroidal and cylindrical grids," Am. J. Phys. 68, 37-40 (2000). 